\title{
Psychological and Physiological Effects of Imagery Rescripting for Aversive Autobiographical Memories
}

\author{
Miriam Strohm ${ }^{1} \cdot$ Marena Siegesleitner ${ }^{1} \cdot$ Anna E. Kunze $^{1} \cdot$ Gabriela G. Werner $^{1} \cdot$ Thomas Ehring $^{1}$. \\ Charlotte E. Wittekind ${ }^{1}$ (i)
}

Accepted: 10 May 2021 / Published online: 25 May 2021

(c) The Author(s) 2021

\begin{abstract}
Background Imagery Rescripting (ImRs) is a promising intervention to reduce symptoms associated with aversive memories, but little is known about its working mechanisms. The present study investigates whether ImRs increases perceived mastery and attenuates emotional reactivity to memory retrieval on a subjective and physiological level.

Methods Seventy-nine individuals reporting memories of distressing real-life events were randomly allocated to ImRs, positive imagery (PI), or no-intervention control (NIC). The memory was reactivated before the intervention and at 1-week follow-up to assess subjective measures and physiological reactivity (heart rate [HR], skin conductance level [SCL], and facial electromyography activity [EMG]) during memory retrieval.

Results ImRs was superior to PI and NIC on subjective memory distress and helplessness, but not on other emotions. ImRs did not exceed PI and NIC in reducing state stress symptoms or increasing perceived mastery. Physiological reactivity (HR, EMG) decreased from pre-intervention to follow-up with no differences between groups.

Conclusions Positive effects of ImRs on helplessness and distress were found. Null findings regarding mastery might result from timing of its assessment or the fact that rather high-functioning participants were included. The lack of between group differences on physiological responses indicates that ImRs did not exceed habituation effects in the present sample.
\end{abstract}

Keywords Imagery rescripting $\cdot$ Mental imagery $\cdot$ Working mechanisms $\cdot$ Psychophysiology $\cdot$ Autobiographical memories

\section{Introduction}

Imagery Rescripting (ImRs) is a promising imagery-based intervention to target aversive memories in emotional disorders (Morina et al., 2017). During ImRs, a memory is first retrieved and emotionally reactivated using mental imagery. In a second step, patients are encouraged to change negative mental images of the distressing event into more positive outcomes according to their individual needs (Arntz, 2012; Holmes et al., 2007). Despite growing evidence for the efficacy of ImRs to reduce symptoms associated with aversive memories in different disorders (for a meta-analysis, see Morina et al., 2017), relatively little is known about the mechanisms underlying symptomatic change.

Charlotte E. Wittekind

charlotte.wittekind@psy.lmu.de

1 Department of Psychology, LMU Munich, Leopoldstraße 13, 80802 Munich, Germany
ImRs has been proposed to work through different mechanisms compared to well-established treatments of aversive emotional memories such as prolonged exposure (PE). Specifically, PE rests on the assumptions of the seminal emotional processing theory (EST; Foa \& Kozak, 1986; Foa et al., 1989; Lang, 1979). The central tenet of EST is that in order to foster emotional processing, the memory representation needs to be activated while new information that is incompatible with the existing memory structure needs to be incorporated (Foa \& Kozak, 1986; Foa et al., 1989). Within-session habituation to fear was regarded as an important indicator that corrective information had been integrated into the memory network (but see Craske et al. [2008] for a review challenging the assumptions underlying emotional processing theory).

ImRs may offer alternative means to first activate aversive memory structures and integrate corrective information (see e.g. Hackmann, 2011). Compared to PE, ImRs induces corrective information more explicitly, for example, by generating mental images of the self as being competent 
and powerful. In this way, ImRs provides new meanings to the aversive memory and targets dysfunctional beliefs typically experienced by patients with trauma-related disorders (e.g. of the self as being incompetent/helpless/incapable; see Foa \& Rothbaum, 1998; Ehlers \& Clark, 2000). Accordingly, Arntz (2012) suggested that ImRs works by activating aversive memories and then providing alternative meanings such that the memory representation of the aversive event is restored with a different and less negative meaning. In consequence, memory-related negative emotional responses should be reduced (Arntz, 2012). There is preliminary evidence supporting the view that ImRs works by changing the meaning of memory representations. In laboratory-based analogue studies, ImRs has been shown to modify the negative valence of memories of aversive film/ pictorial stimuli and to reduce associated negative emotional responding (Dibbets et al., 2012, 2018; Hagenaars \& Arntz, 2012). Moreover, there is evidence that ImRs might specifically work by fostering perceived mastery in face of aversive experiences. Participants who had experienced distressing life-events appraised the originally distressing experience as being more controllable after ImRs indicating that ImRs had led to a revaluation of the aversive autobiographical memory contents (Strohm et al., 2019). The beneficial effects of ImRs on perceived mastery may be due to the fact that during ImRs individuals are encouraged to actively change the sequence of events according to their individual needs and to express action tendencies that had been inhibited in the original situation, for example, defending oneself and disempowering the perpetrator (Arntz, 2012). In patients with nightmare disorder, perceived mastery (of the nightmare content) mediated the beneficial effects of ImRs. Importantly, this mediation only emerged in the ImRs condition and was not observed in individuals receiving imaginal exposure treatment (Kunze, Arntz, et al., 2019; Kunze, Lancee, et al., 2019). This indicates that enhancing mastery might be a working mechanism that is specific for ImRs.

In addition, there is evidence that ImRs attenuates negative emotional responding to autobiographical memories. Specifically, ImRs reduced negative emotions and distress triggered by memories of aversive life-events that were retrieved following ImRs (Cili et al., 2016; Nilsson et al., 2012; Reimer \& Moscovitch, 2015; Strohm et al., 2019). One limitation of the latter studies is that emotional responding to the memories was only assessed on a subjective level. If ImRs indeed modifies memory representations of distressing events such that the memory is restored with a less negative meaning, memory-related physiological responding to the memory should also be reduced (Arntz, 2012). However, until now only few studies have included physiological outcome measures in the context of ImRs research.

Findings from recent experimental studies using fear conditioning paradigms are inconclusive with respect to the effects of ImRs on physiological responding to conditioned stimuli (Dibbets et al., 2012, 2018; Kunze, Arntz, et al., 2019; Kunze, Lancee, et al., 2019). In participants with increased health anxiety, ImRs (as well as imagery reexperiencing) yielded higher physiological activation (heart rate [HR]) during the intervention compared to a positive imagery and a no intervention control condition (Tolgou et al., 2018). Moreover, there was a trend for ImRs yielding the greatest HR reductions during the intervention, possibly indicating successful emotional processing (Tolgou et al., 2018). In patients with social anxiety disorder (SAD), ImRs was found to selectively attenuate physiological reactivity (HR parameters) to a speech task compared with verbal restructuring and a waitlist control condition, but the ImRs group was more reactive to the speech task after treatment indexed by skin conductance (Hyett et al., 2018). Taken together, there is preliminary evidence that ImRs leads to physiological activation during the intervention and reduces physiological responding to stressors. However, it has not yet been examined whether ImRs attenuates the physiological reactivity to aversive autobiographical memories retrieved after treatment.

The aim of the present study was twofold: First, we aimed to replicate previous findings that ImRs increases perceived mastery (Kunze, Arntz, et al., 2019; Kunze, Lancee, et al., 2019; Strohm et al., 2019) and reduces self-reported negative emotional responses to memory retrieval (Cili et al., 2016; Nilsson et al., 2012; Reimer \& Moscovitch, 2015; Strohm et al., 2019). Second, we aimed to extend previous research by examining whether ImRs changes physiological responding to memories of aversive life-events.

The present study was based on an experimental paradigm used by Strohm et al. (2019). In this earlier study, the effects of ImRs versus a no intervention control group (NIC) were examined in healthy individuals reporting aversive memories of distressing real-life events. In the current study, the design of this study was extended by including (a) physiological measurements in addition to subjective reports and (b) a positive imagery condition (PI) as an active control condition to control for the effects of activating a memory representation in combination with inducing positive mental images, emotions, and meanings, but without changing meaning-relevant contents of the original aversive memory. First, we expected that ImRs would lead to stronger reductions of subjective distress associated with the aversive lifeevent and of stress symptoms elicited by memory retrieval compared to the control conditions (Hypothesis 1). Second, in line with the proposed working mechanism and based on previous findings, ImRs was expected to be superior to PI and NIC in enhancing perceived mastery (Hypothesis 2) and in decreasing negative emotions, distress, and arousal at memory retrieval (Hypothesis 3). Finally, we assumed that ImRs would lead to stronger reductions in physiological 
arousal (Hypothesis 4), indexed by HR and skin conductance level (SCL), as well as negative emotional valence, indexed by facial electromyography activity (EMG), at memory retrieval.

\section{Method}

\section{Participants}

Individuals who had experienced a distressing life-event in the past 24 months (e.g. relationship break-up, job loss, bullying, interpersonal conflicts) were recruited via social media, online newsletter of the university, and notices on university campuses. Participants had to meet the following inclusion criteria: (1) experience of a distressing but non-traumatic life-event (i.e. not meeting criterion A of DSM-5, APA, 2013) within the past 24 months, (2) subjective distress of at least 50 (Subjective Units of Distress [SUD] on a 0-100 scale, ranging from not at all distressed to extremely distressed) at the time the event happened, (3) distress of at least 30 (on the same $0-100$ scale) at the time of study participation (see Table 1 for average past and current distress), and (4) recurrent distressing memories of the life-event in the form of (a) intrusive thoughts or images, (b) nightmares, or (c) emotional/physiological responding to reminders of the event. Individuals reporting a case of death as their life-event were excluded due to ethical concerns. We also excluded individuals meeting the following criteria: (1) current diagnosis of a mental disorder (including acute suicidal tendencies), (2) lifetime diagnosis of PTSD/psychotic disorder/ bipolar disorder, (3) psychological treatment at the time of study participation, (4) severe physical illness, (5) pregnancy, and (6) age below 18 or above 35 years. All inclusion and exclusion criteria were assessed in Session 1 using a short structured screening interview and the German version of the Mini International Neuropsychiatric Interview for DSM-5 (M.I.N.I. 7.0.2; Sheehan et al., 1998; Sheehan, 2016). Fifteen individuals had to be excluded ( $n=7$ reported a life-event not meeting inclusion criteria; $n=7$ met criteria for a current or lifetime diagnosis of the mental disorders specified above, $n=1$ technical problems), leaving a total sample of 79 students included in this study (age: $M=24.20$, $S D=3.84 ; 83.5 \%$ female). Ethical approval was obtained from the Ethics Committee at LMU Munich. Participants received either partial course credit or $22 €$. All participants gave written informed consent.

\section{Memory Reactivation Task}

In both sessions, a memory reactivation task was used to measure self-reported and physiological responses to memory retrieval. First, the most distressing "scene" of the lifeevent was determined in order to get a specific memory for the memory reactivation task (and subsequently for ImRs). Additionally, participants were asked to indicate the most aversive moment within this "scene" to specify the individual hotspot. The memory reactivation task comprised

Table 1 Sample Characteristics and Baseline Comparisons

\begin{tabular}{|c|c|c|c|c|c|}
\hline & Overall Sample $(N=79)$ & $\begin{array}{l}\text { ImRs } \\
(n=27)\end{array}$ & $\begin{array}{l}\text { PI } \\
(n=25)\end{array}$ & $\begin{array}{l}\mathrm{NIC} \\
(n=27)\end{array}$ & Comparison between conditions \\
\hline Gender ( $n$ female/male) & $66 / 13$ & $22 / 5$ & $22 / 3$ & $22 / 5$ & $\chi^{2}=0.59, p=.802$ \\
\hline Age in years: $M(S D)$ & $24.20(3.84)$ & $23.96(4.02)$ & $23.24(3.11)$ & $25.33(4.11)$ & $F(2,76)=2.06, p=.135$ \\
\hline $\begin{array}{l}\text { Time since life-event (in months): } M \\
\quad(S D)\end{array}$ & $7.67(6.52)$ & $6.85(5.44)$ & $8.87(6.94)$ & $7.37(7.15)$ & $F(2,76)=0.66, p=.520$ \\
\hline SUD time of the event: $M(S D)$ & $87.66(9.25)$ & $88.63(8.22)$ & $84.88(10.00)$ & $89.26(9.27)$ & $F(2,76)=1.71, p=.188$ \\
\hline $\begin{array}{l}\text { SUD beginning of study participation: } \\
\qquad M(S D)\end{array}$ & $51.98(18.46)$ & $52.22(17.06)$ & $48.60(18.12)$ & $54.89(20.21)$ & $F(2,76)=0.75, p=.475$ \\
\hline \multicolumn{6}{|l|}{ Categories of the distressing life-events } \\
\hline Relationship difficulties or break-ups & $54 \%$ & $59 \%$ & $52 \%$ & $55 \%$ & \\
\hline Family conflicts & $10 \%$ & $7 \%$ & $12 \%$ & $8 \%$ & \\
\hline Problems at work, bullying & $9 \%$ & $11 \%$ & $4 \%$ & $11 \%$ & \\
\hline Problems at university & $8 \%$ & $0 \%$ & $16 \%$ & $8 \%$ & \\
\hline Accidents & $8 \%$ & $11 \%$ & $4 \%$ & $8 \%$ & \\
\hline Serious illness of a close person & $3 \%$ & $0 \%$ & $4 \%$ & $3 \%$ & \\
\hline Other events & $8 \%$ & $11 \%$ & $8 \%$ & $8 \%$ & \\
\hline $\begin{array}{l}\text { Depressive symptoms (PHQ-9): } M \\
\quad(S D)\end{array}$ & $15.72(4.72)$ & $14.30(4.52)$ & $15.76(4.20)$ & $17.11(5.10)$ & $F(2,76)=2.49, p=.089$ \\
\hline
\end{tabular}

ImRs Imagery Rescripting; PIPositive Imagery; NIC No-Intervention Control Condition; SUD Subjective Units of Distress; $P H Q-9$ Patient Health Questionnaire-9 
two phases: During Phase 1 (termed narrative imagination in Fig. 1 and hereinafter), participants were instructed to close their eyes and to vividly imagine the specified memory focusing on all sensory modalities and emotions. They were asked to describe the event as if it was happening right now in the first person perspective and in present tense. Phase 1 of memory reactivation ended with the participant imagining the individual hotspot (mean duration of Phase 1 was $3.96 \min [S D=1.64]$ in Session 1 and $3.13 \min [S D=0.71]$ in Session 2). Participants were then administered self-report measures on responses to memory retrieval (see section 'Subjective Responses to Memory Retrieval'). In Phase 2 of the memory reactivation (termed hotspot imagination in Fig. 1 and hereinafter), participants were instructed to close their eyes and to vividly imagine the hotspot of their memory for one minute (without talking) and to be aware of upcoming emotions. This phase was used for an additional recording of physiological responses to memory retrieval without talking, given that physiological reactions have been shown to be influenced by changes in respiration patterns during speech (see Beda et al., 2007; Quintana \& Heathers, 2014).

\section{Experimental Conditions}

\section{Imagery Rescripting}

During ImRs, participants were guided by means of a semistructured ImRs protocol. The first phase of the ImRs protocol closely followed the procedure suggested by Arntz and Weertman (1999) in that participants were instructed to vividly imagine the aversive event as vividly and detailed as possible and if it was happening at the moment. To achieve affective activation, participants were instructed to describe the event in present tense, from a first person perspective, and to focus on all sensory modalities, feelings, and body sensations. In the second phase of ImRs, however, participants were not instructed to switch perspective as usually done in clinical practice with patients with early traumatic memories (phase 2: adult self is entering the image and intervenes; phase 3 : interventions by the adult experienced from the child's perspective), as life-events were recent in our sample. ImRs was administered by a female experimenter with a Bachelor's degree in psychology, who was continuously trained and supervised by the first author, who has had extensive training in ImRs (i.e. multiple-day workshops by A. Arntz and M. Smucker, for more information on the training procedure see Supplementary Material).

The first phase of the intervention aimed to reactivate memory-related emotions (duration: $M=3.41 \mathrm{~min}$, $S D=1.15)$ and it ended with the participant holding the image of the individual hotspot in mind. The rescripting of the memory (duration: $M=11.26 \mathrm{~min}, S D=2.97$ ) was then initiated and participants were asked to change the course of events in any desired way to make it less distressing (changes could be realistic or unrealistic). They were encouraged to imagine the new script as vividly as possible and to describe it out loud in detail. During the rescripting of the memory, the following questions were asked to support participants (see Arntz \& Weertman, 1999): What would you like to do/ say? Ok, do it/say it! What do you feel? What do you think? Is there anything else you would like to change? Is there anything (else) you need?. When participants did not wish to include further changes but were satisfied with the new outcome of the event, they were asked to stay with the final positive image for a moment before concluding the imagery exercise.

\section{Positive Imagery}

Participants were asked to vividly imagine a positive event of the past 24 months and to describe it to the experimenter in the first person perspective, present tense, focusing on body sensations and emotions (duration: $M=9.20 \mathrm{~min}$, $S D=1.45$ ). The positive event had to be unrelated to the distressing life-event. Participants rated the positive events as highly pleasant (time of the event: $M=93.64, S D=8.44$; time of study participation: $M=89.00, S D=11.55 ; 0-100$

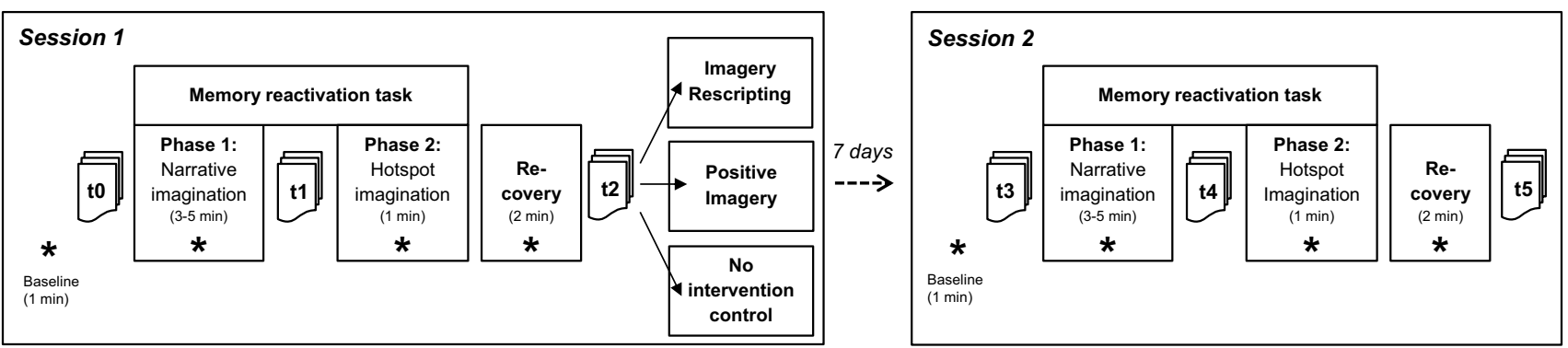

Fig. 1 Schematic Overview of the Study Procedure. * = Physiological Measurement phases; t0: Sociodemographic Data, PHQ, EventRelated Distress, Emotions, Self-Reported Arousal; t1: Emotions,
Self-Reported Arousal, Memory Distress, Mastery; t2: RSDI; t3: Event-Related Distress, Emotions, Self-Reported Arousal; t4: Emotions, Self-Reported Arousal, Memory Distress, Mastery; t5: RSDI 
scale, ranging from not at all pleasant to extremely pleasant).

\section{No-Intervention Control Condition}

Participants were provided neutral magazines and were asked to wait $20 \mathrm{~min}$ in the laboratory.

\section{Self-Report Measures}

\section{Depressive Symptoms}

The Patient Health Questionnaire-9 Item (PHQ-9; Krönke et al., 2001; German version: Löwe et al., 2002) was administered to test for baseline differences in depressive symptoms. The PHQ-9 is a reliable and valid measure of depression severity (Krönke et al., 2001; internal consistency in the present study: Cronbach's $\alpha=0.83$ ).

\section{Event-Related Distress}

In order to assess intervention effects on distress related to the life-event, we asked participants how distressed they felt by the life-event during the past week on a 100 -mm visual analogue scale (VAS) ranging from 0 (not at all distressed) to 100 (extremely distressed).

The Response to Script Driven Imagery Scale (RSDI; Hopper et al., 2007) was used to measure state stress symptoms evoked by mental imagery of the aversive memories. All items referred to the imagination (Instruction: "Please go back to the imagination during which you imagined the distressing event. During the imagination...”.). Stress symptoms were assessed on three subscales: re-experiencing (4 items, e.g. "Did you feel as though the event was reoccurring, like you were reliving it?", "Were you emotionally upset?"), avoidance (3 items, e.g. "Did you avoid thoughts about the event?"), and dissociation (3 items, e.g. "Did you feel disconnected from your body?"), with a scale ranging from 0 (not at all) to 6 (a great deal). In the present study instructions of the RSDI were adapted to measure responses to the memory reactivation task, which was not scriptdriven. Mean subscale scores were computed to facilitate comparison between scales (see Hopper et al., 2007). The RSDI has demonstrated good psychometric properties (Hopper et al., 2007; internal consistencies in the present study: Cronbach's $\alpha \mathrm{s}=0.59-0.83$ ).

\section{Subjective Responses to Memory Retrieval}

Mastery After memory reactivation, perceived mastery was assessed by asking participants to verbally indicate how controllable they experienced the situation that they had just imagined, on a scale ranging from 0 (not at all controllable) to 100 (very controllable).

Emotions In order to assess the effects of the memory reactivation on emotions five negative (anxious, angry, sad, guilty, helpless) and three positive emotions (happy, satisfied, proud) were assessed on 100-mm VAS administered before and after the memory reactivation (see Fig. 1). Participants were instructed to indicate how they felt at the moment on a scale ranging from 0 (not at all) to 100 (extremely). The distinct emotions were presented in the same order in both sessions (as listed above).

Self-Reported Arousal Subjective physiological arousal was assessed after memory reactivation (and at baseline) using self-assessment manikins (SAM; Bradley \& Lang, 1994). On a scale ranging from 1 (very calm) to 9 (very aroused) participants were asked how aroused they felt at the moment.

Memory Distress Memory distress was assessed verbally after memory reactivation using Subjective Units of Distress (SUD). Participants were asked to indicate how distressed they felt at the moment on a scale ranging from 0 (not at all distressed) to 100 (extremely distressed).

\section{Physiological Measurement}

Physiological activity was recorded using a stationary system for psychophysiological measurements (Refa; Twente Medical Systems International [TMSi], EJ Oldenzaal, The Netherlands) and the recording software package Polybench 1.30 (TMSi). The sampling rate was $1024 \mathrm{~Hz}$. A wet band on the left wrist served as grounding for all channels.

We used electrocardiography (ECG) to measure cardiovascular activity (HR in beats per minute [bpm]). ECG electrodes were applied on the upper sternum and lowest rib on the left side. A $0.05 \mathrm{~Hz}$ highpass filter was applied during ECG measurement. Raw data were bandpass filtered between 0.5 and $40 \mathrm{~Hz}$ (Wilhelm et al., 1999). For further processing, the software Autonomic Nervous System Laboratory (ANSLAB) 2.6 (Blechert et al., 2016) was used to automatically determine R-spikes, which were subsequently manually checked. HR was assessed as an index for physiological arousal (Mauss \& Robinson, 2009).

Electrodermal activity (i.e. SCL) was obtained by applying a constant voltage $(0.5 \mathrm{~V})$ between the index and middle finger of the non-dominant hand, using an isolated electrodermal amplifier module supplied by Becker Meditec (Karlsruhe, Germany) and $\mathrm{Ag} / \mathrm{AgCl}$ electrodes with 5-mm inner-diameter, filled with isotonic paste (TD-246, MedCat, Germany). Mean SCL in $\mu \mathrm{S}$ was computed and 
fluctuations with an increase larger than $0.02 \mu \mathrm{S}$ were considered a response. SCL was also assessed as an index for physiological arousal (Mauss \& Robinson, 2009).

For EMG, facial muscle activity of the $m$. corrugator supercilii was assessed by one pair of 2-mm inner-diameter $\mathrm{Ag} / \mathrm{AgCl}$ electrodes, that were placed with centers approximately $1 \mathrm{~cm}$ apart above the participant's right eye-brow (Fridlund \& Cacioppo, 1986). EMG preprocessing using ANSLAB 2.6 comprised a $28 \mathrm{~Hz}$ high-pass filter, $50 \mathrm{~Hz}$ notch filter, rectification, low pass filtering $(15.92 \mathrm{~Hz})$ and $50 \mathrm{~ms}$ moving average filter. Facial muscle activity of the $m$. corrugator supercilii was used as a measure of the valence of participants' emotional states during memory reactivation (see Mauss \& Robinson, 2009).

Recorded data were further processed using the software ANSLAB version 2.6 (Blechert et al., 2016). Physiological activity before the memory reactivation task (1 min resting baseline), during memory reactivation (Phase 1: narrative imagination; Phase 2: hotspot imagination), and after memory reactivation ( $2 \mathrm{~min}$. recovery phase) was included (as results of the recovery phase were exploratory, they are presented in the Supplementary Material). For each of these experimental periods, mean scores of HR, SCL, and EMG were computed.

\section{Procedure}

The study consisted of two sessions with seven days in between the sessions. Both sessions took place at the same time of the day. Questionnaires were administered digitally (unless otherwise specified in the section Self-Report Measures) using the online platform Unipark ${ }^{\circledR}$ and Eprime. For an overview of the study procedure see Fig. 1.

\section{Session 1}

Screening interviews were administered to assess inclusion and exclusion criteria. After study inclusion, electrodes for physiological measurements were attached and participants then filled out baseline questionnaires ( $\mathrm{t} 0$ : sociodemographic data, PHQ, event-related distress, emotions, self-reported arousal) and were provided a demonstration of an imagery exercise by the experimenter (imagination of today's breakfast). This was followed by a $1 \mathrm{~min}$ baseline assessment of HR, SCL, and EMG. Next, the memory reactivation task was administered that included self-report measures referring to memory reactivation Phase 1 ( $\mathrm{t} 1$ : mastery, emotions, self-reported arousal, memory distress) and the two phases of physiological measurement described above. After memory reactivation, participants were instructed to relax for two minutes (recovery phase), followed by the RSDI (t2). After a 10 min break, participants were randomly allocated to ImRs $(n=27)$, PI $(n=25)$, or NIC $(n=27)$ using a computer-generated allocation sequence. Groups were stratified by gender. The experimenter was blind to condition until the beginning of the interventions.

\section{Session 2}

Participants first filled out baseline questionnaires (t3: eventrelated distress, emotions, self-reported arousal), followed by a $1 \mathrm{~min}$ baseline assessment of HR, SCL and EMG. Next, the memory reactivation task was administered using the same memory and the same procedure as in Session 1 (including t4: mastery, emotions, self-reported arousal, memory distress, and physiological measurement Phases 1 and 2). After a 2 min recovery phase, participants filled out the RSDI (t5). Finally, participants were fully debriefed.

\section{Data Preparation}

For emotions and self-reported arousal, reactivity scores (Session 1: t1-t0; Session 2: t4-t3) were computed. For physiological measures, reactivity and recovery indices were computed for both sessions (see Hyett et al., 2018). Reactivity was defined as the difference between baseline physiological activity and activity during the first minute of memory reactivation Phase 1 (narrative imagination; reactivity $=$ mean memory reactivation Phase 1 -mean baseline) or Phase 2 (hotspot imagination; reactivity = mean memory reactivation Phase 2-mean baseline), respectively.

\section{Statistical Analyses}

A manipulation check with reactivity scores of Session 1 was carried out to test effects of the memory reactivation task on subjective and physiological responses using 2 (Time) $\times 3$ (Condition) repeated measures analyses of variance (ANOVAs) for self-reported arousal, HR, SCL, EMG and two repeated measures multivariate analyses of variance (MANOVAs) for negative and positive emotions as dependent variables.

Main analyses for event-related distress, memory distress, mastery, and RSDI, comprised a series of 2 (Time) $\times 3$ (Condition) repeated measures ANOVAs. Hypotheses regarding emotions and self-reported arousal were tested using 2 (Time) $\times 3$ (Condition) repeated measures ANOVAs with reactivity scores of Session 1 and Session 2 as dependent variables. A series of 2 (Time) $\times 3$ (Condition) repeated measures ANOVAs was conducted for reactivity scores of HR, SCL, and EMG. For self-report and physiological measures, significant interaction effects were followed up using planned contrasts on change scores (Session 2 - Session 1) to test for differences between ImRs and the control conditions (ImRs vs. PI+ NIC) as well as between ImRs and PI. 
Partial eta squared $\left(\eta_{\mathrm{p}}^{2}\right)$ or Cohen's $d$ were used as effect sizes and criterion of significance was set at $\alpha=0.05$ (twosided) for all analyses.

Due to technical problems, physiological data of three participants were not recorded ( $n=1 \mathrm{ImRs} ; n=2 \mathrm{NIC})$ and data for single measurement phases were missing for four participants in Session 1 and one participant in Session 2. Self-report measures were erroneously not administered in some participants ( $n=1$ for RSDI, emotions, arousal; $n=2$ for event-related distress). These participants were excluded from the respective analyses.

\section{Results}

\section{Sample Characteristics and Baseline Comparisons}

There were no significant differences between conditions on sociodemographic and baseline measures (see Table 1).

\section{Manipulation Check}

For self-reported arousal, there was an overall increase from pre- to post-memory reactivation (t0 vs. t1) during Session $1, F(1,75)=183.35, p<0.001, \eta_{\mathrm{p}}^{2}=0.71$, with no differences between conditions, all $F \mathrm{~s}(2,75)<0.60, p \mathrm{~s}>0.552$, $\eta_{p}^{2} s<0.02$. Levels of negative emotions increased from preto post-memory reactivation ( $\mathrm{t} 0 \mathrm{vs} . \mathrm{t} 1$ ), whereas levels of positive emotions decreased, all $F \mathrm{~s}(5,71 / 3,73)>33.39$, $p \mathrm{~s}<0.001, \eta_{\mathrm{p}}^{2} \mathrm{~s}>0.70$, with no differences between conditions, all $F \mathrm{~s}(10,144 / 6,148)<1.03, p \mathrm{~s}>0.419, \eta_{\mathrm{p}}^{2} \mathrm{~s}<0.07$ (see Table 2).

The manipulation check for physiological measures revealed an increase in HR from baseline to both phases of memory reactivation, all $F \mathrm{~s}(1,71 / 1,72)>13.48$, $p \mathrm{~s}<0.001, \eta_{\mathrm{p}}^{2} \mathrm{~s}>0.16$. Additionally, groups differed significantly in their overall HR levels, all $F \mathrm{~s}(2,71 / 2,72)>6.48$, $p s<0.003, \eta_{p}^{2} s>0.15$, with ImRs participants showing the highest mean HR across time points. However, there were no significant Time $\mathrm{x}$ Condition interaction effects, all $F \mathrm{~s}(2,71 / 2,72)<2.48, p \mathrm{~s}>0.091, \eta_{\mathrm{p}}^{2} \mathrm{~s}<0.06$. While SCL increased during Phase 1 of the memory reactivation (narrative imagination), we unexpectedly found reductions in SCL from baseline to Phase 2 (hotspot imagination), all $F_{\mathrm{s}}(1,71$ $/ 1,72)>4.43, p s<0.039, \eta_{\mathrm{p}}^{2} \mathrm{~s}>0.06$, with no differences between groups, all $F \mathrm{~s}(2,72 / 2,71)<1.35, p s>0.267$; $\eta_{\mathrm{p}}^{2} \mathrm{~s}<0.04$. EMG activity only increased in response to memory reactivation Phase $2, F(1,71)=23.12, p<0.001$, $\eta_{\mathrm{p}}^{2}=0.25$, but not in response to memory reactivation Phase $1, F(1,71)=0.27, p=0.605, \eta_{\mathrm{p}}^{2}<0.01$, with no differences between groups, all $F \mathrm{~s}(2,71)<0.89, p \mathrm{~s}>0.416 ; \eta_{\mathrm{p}}^{2} \mathrm{~s}<0.02$.
Table 2 Means and Standard Deviations of Emotional Reactivity to the Memory Before (Session 1; t1-t0) and After the Interventions (Session 2; t4-t3)

\begin{tabular}{|c|c|c|c|}
\hline & Group & $\begin{array}{l}\text { Session } 1 \\
M(S D)\end{array}$ & $\begin{array}{l}\text { Session } 2 \\
M(S D)\end{array}$ \\
\hline \multicolumn{4}{|c|}{ Negative emotions } \\
\hline \multirow[t]{3}{*}{ Anxious } & $\operatorname{ImRs}$ & $22.69(25.36)$ & $9.23(16.59)$ \\
\hline & PI & $20.24(18.40)$ & 9.97 (23.89) \\
\hline & NIC & $11.32(20.53)$ & $10.46(22.34)$ \\
\hline \multirow[t]{3}{*}{ Angry } & $\operatorname{ImRs}$ & $32.89(32.95)$ & $18.25(22.54)$ \\
\hline & PI & $26.86(25.20)$ & $19.98(20.62)$ \\
\hline & NIC & $31.74(40.16)$ & $28.73(31.95)$ \\
\hline \multirow[t]{3}{*}{$\mathrm{Sad}$} & $\operatorname{ImRs}$ & $41.27(31.02)$ & $23.80(23.86)$ \\
\hline & PI & $32.69(23.11)$ & $26.84(20.57)$ \\
\hline & NIC & $30.38(23.12)$ & $27.73(24.45)$ \\
\hline \multirow[t]{3}{*}{ Guilty } & ImRs & $19.74(29.49)$ & $14.54(19.57)$ \\
\hline & PI & 22.74 (31.99) & $12.45(23.87)$ \\
\hline & NIC & $19.85(23.97)$ & $14.81(20.22)$ \\
\hline \multirow[t]{3}{*}{ Helpless } & ImRs & $42.11(32.66)$ & $21.52(25.79)$ \\
\hline & PI & 33.55 (27.28) & $30.44(24.30)$ \\
\hline & NIC & $25.55(29.00)$ & $30.51(29.74)$ \\
\hline \multicolumn{4}{|c|}{ Positive emotions } \\
\hline \multirow[t]{3}{*}{ Happy } & ImRs & $-32.43(18.24)$ & $-18.74(15.24)$ \\
\hline & PI & $-30.87(14.86)$ & $-20.46(14.00)$ \\
\hline & NIC & $-27.68(17.51)$ & $-22.54(13.37)$ \\
\hline \multirow[t]{3}{*}{ Satisfied } & $\operatorname{ImRs}$ & $-32.41(18.77)$ & $-20.97(15.97)$ \\
\hline & PI & $-31.22(21.28)$ & $-22.10(18.44)$ \\
\hline & NIC & $-28.57(20.64)$ & $-22.78(15.63)$ \\
\hline \multirow[t]{3}{*}{ Proud } & ImRs & $-18.59(24.16)$ & $-9.03(19.20)$ \\
\hline & PI & $-14.08(27.12)$ & $-12.31(20.48)$ \\
\hline & NIC & $-23.10(22.66)$ & $-11.20(16.05)$ \\
\hline \multicolumn{4}{|l|}{ Arousal } \\
\hline \multirow[t]{3}{*}{ SAM } & ImRs & $2.81(1.47)$ & $1.69(1.16)$ \\
\hline & PI & $2.84(2.12)$ & $2.20(1.76)$ \\
\hline & NIC & $2.37(1.60)$ & $2.11(1.40)$ \\
\hline
\end{tabular}

ImRs Imagery Rescripting; PIPositive Imagery; NIC No-Intervention Control Condition; SAM Self-Assessment Manikin

Therefore, only the EMG reactivity score of Phase 2 (hotspot imagination) was included in the main analyses.

\section{Subjective Outcomes}

\section{Event-Related Distress and Stress Symptoms (Hypothesis 1)}

Conditions differed in baseline to follow-up changes of distress related to the life-event as indicated by a significant Time $\mathrm{x}$ Condition interaction, $F(2,74)=4.26, p=0.018$, $\eta_{\mathrm{p}}^{2}=0.10$. Planned contrasts revealed that ImRs led to stronger reductions in distress from Session 1 to Session 2 compared to the control conditions (PI + NIC), $t(74)=-2.80$, 
a

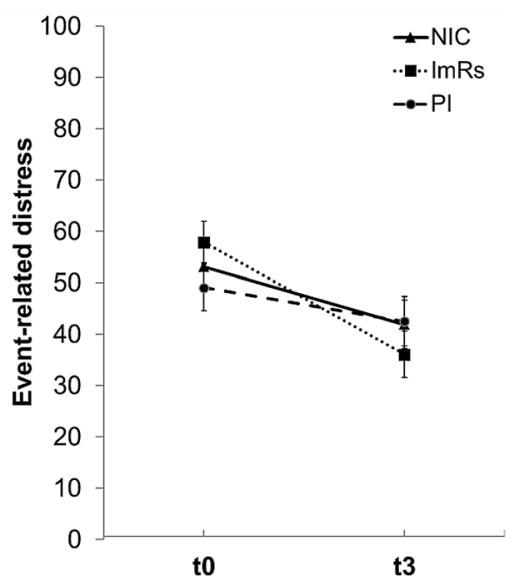

b

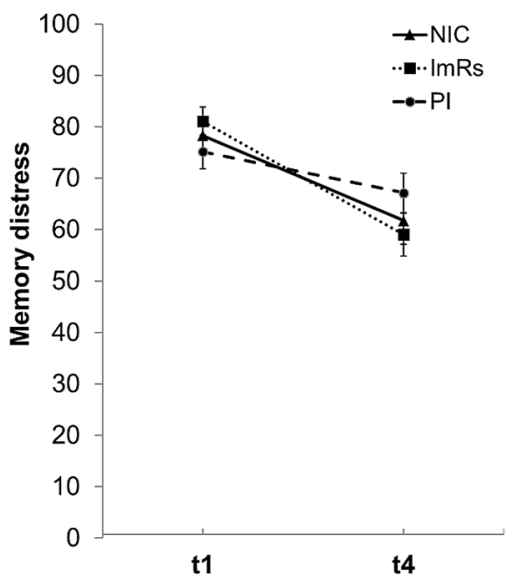

c

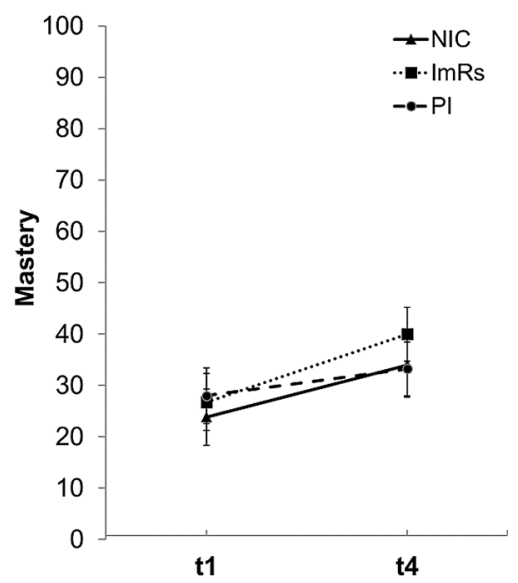

Fig. 2 Effects of Imagery Rescripting (ImRs) vs. Positive Imagery (PI) vs. No-Intervention Control Condition (NIC) on a Distress Related to the Life-Event During the Last Week, b Memory Distress, and c Mastery; $\mathrm{t} 0, \mathrm{t} 1$ : Before the Interventions (Session 1); $\mathrm{t} 3, \mathrm{t} 4$ : After the Interventions (Session 2); Error Bars Represent SEM

significant main or interaction effects were observed, all $F_{\mathrm{s}}(1,75 / 2,75)<2.35, \mathrm{ps}>0.129, \eta_{\mathrm{p}}^{2} \mathrm{~s}<0.03$.

3 Means and Standard Deviations of Stress Symptoms Durin Memory Reactivation Before (Session 1) and After the Interventions (Session 2)

\begin{tabular}{llll}
\hline & Group & $\begin{array}{l}\text { Session 1 } \\
M(S D)\end{array}$ & $\begin{array}{l}\text { Session 2 } \\
M(S D)\end{array}$ \\
\hline RSDI & & & \\
Re-experiencing & ImRs & $4.29(0.68)$ & $3.44(1.26)$ \\
& PI & $4.34(1.01)$ & $3.81(1.01)$ \\
Avoidance & NIC & $4.57(1.04)$ & $3.69(1.24)$ \\
& ImRs & $1.72(1.37)$ & $2.13(1.42)$ \\
& PI & $2.12(1.34)$ & $2.24(1.41)$ \\
Dissociation & NIC & $1.79(1.41)$ & $2.12(1.49)$ \\
& ImRs & $1.89(1.18)$ & $2.21(1.42)$ \\
& PI & $1.96(1.05)$ & $2.13(1.16)$ \\
& NIC & $1.71(1.24)$ & $1.80(1.48)$ \\
\hline
\end{tabular}

$R S D I$ Response to Script Driven Imagery Scale; ImRs Imagery Rescripting; PIPositive Imagery; NIC No-Intervention Control Condition

$p=0.007, d=0.68$, with ImRs yielding stronger decreases than PI, $t(74)=2.84, p=0.006, d=0.80$ (see Fig. 2).

Regarding stress symptoms, results of the re-experiencing subscale of the RSDI (see Table 3 ) revealed that symptoms were reduced from Session 1 to Session $2, F(1,75)=32.81$, $p<0.001, \eta_{\mathrm{p}}^{2}=0.30$, but neither an interaction with condition nor a main effect of condition could be observed, all $F \mathrm{~s}(2,75)<0.71, p \mathrm{~s}>0.496, \eta_{\mathrm{p}}^{2} \mathrm{~s}<0.02$. Avoidance symptoms increased over time, $F(1,75)=3.98, p=0.050$, $\eta_{\mathrm{p}}^{2}=0.05$, again no interaction with condition and no significant main effect of condition emerged, $F \mathrm{~s}(2,75)<0.35$, $p s>0.703, \eta_{p}^{2} \mathrm{~s}<0.01$. For the dissociation subscale no

\section{Mastery (Hypothesis 2)}

Mastery increased from Session 1 to Session 2, $F(1$, $76)=14.62, p<0.001, \eta_{p}^{2}=0.16$, but neither an interaction with condition nor a main effect of condition could be observed, all $F_{\mathrm{s}}(2,76)<0.85, p>0.432, \eta_{\mathrm{p}}^{2} \mathrm{~s}<0.02$ (see Fig. 2).

\section{Negative Emotions, Memory Distress, and Self-Reported Arousal (Hypothesis 3)}

Pre- and posttreatment reactivity scores per condition are provided in Table 2 (for raw scores see Table S1 in the Supplementary Material). For helplessness, changes in reactivity differed significantly between groups as indicated by a significant Time $\mathrm{x}$ Condition interaction, $F(2,75)=5.14$, $p=0.008, \eta_{\mathrm{p}}^{2}=0.12$. Planned contrasts showed that ImRs reduced helplessness to the memory more strongly than the control conditions, $t(75)=-3.05, p=0.003, d=0.73$; ImRs also led to stronger decreases in helplessness than PI, $t(75)=-2.12, p=0.037, d=0.59$. Besides helplessness, sadness appeared to be a relevant emotion; however, the interaction of Time $\mathrm{x}$ Condition for reactivity scores was not significant, $F(2,75)=2.03, p=0.138, \eta_{\mathrm{p}}^{2}=0.05$. Given the medium effect, for exploratory reasons, contrasts were calculated and revealed that ImRs led to a stronger reduction of sadness than the control conditions, $t(75)=-1.97$, $p=0.052, d=0.47$; however, the difference between ImRs and PI was not significant, $t(75)=-1.49, p=0.142, d=0.41$. For anxiety, anger, and guilt, reductions of emotions to the 
memory could be observed from Session 1 to Session 2, all $F \mathrm{~s}(1,75)>4.49, p \mathrm{~s}<0.037, \eta_{\mathrm{p}}^{2} \mathrm{~s}>0.06$, but neither an interaction with condition nor a main effect of condition could be observed, all $F \mathrm{~s}(2,75)<2.29, p \mathrm{~s}>0.109, \eta_{\mathrm{p}}^{2} \mathrm{~s}<0.06$.

Changes in memory distress differed between groups as indicated by a significant Time $\mathrm{x}$ Condition interaction effect, $F(2,76)=3.36, p=0.040, \eta^{2}{ }_{p}=0.08$. Planned contrasts showed that ImRs reduced memory distress more strongly from Session 1 to Session 2 compared to the control conditions (PI + NIC), $t(76)=-2.09, p=0.040$, $d=0.50$, and ImRs was superior to PI, $t(76)=2.58$, $p=0.012, d=0.72$ (see Fig. 2)

For self-reported arousal (see Table 2), reductions in reactivity scores were observed from Session 1 to Session $2, F(1,75)=11.19, p=0.001, \eta_{p}^{2}=0.13$, but neither an interaction with condition nor a main effect of condition could be observed, all $F \mathrm{~s}(2,75)<1.55, p \mathrm{~s}>0.219$, $\eta_{\mathrm{p}}^{2} \mathrm{~s}<0.04$.

Table 4 Means and Standard Deviations of Physiological Reactivity Scores Before (Session 1) and After ImRs (Session 2)

\begin{tabular}{|c|c|c|c|}
\hline & Group & $\begin{array}{l}\text { Session } 1 \\
M(S D)\end{array}$ & $\begin{array}{l}\text { Session } 2 \\
M(S D)\end{array}$ \\
\hline \multicolumn{4}{|c|}{ Reactivity (Narrative imagination) } \\
\hline \multirow[t]{3}{*}{$\mathrm{HR}$} & $\operatorname{ImRs}$ & $13.60(8.54)$ & $8.02(6.49)$ \\
\hline & PI & $16.06(12.29)$ & $11.24(7.82)$ \\
\hline & NIC & $15.64(8.79)$ & $11.51(8.04)$ \\
\hline \multirow[t]{3}{*}{ SCL } & ImRs & $0.18(0.38)$ & $0.86(0.85)$ \\
\hline & PI & $0.24(0.64)$ & $0.75(0.95)$ \\
\hline & NIC & $0.13(1.04)$ & $0.78(0.90)$ \\
\hline \multirow[t]{3}{*}{ EMG } & $\operatorname{ImRs}$ & $0.14(3.23)$ & $-1.25(2.34)$ \\
\hline & PI & $-0.36(2.34)$ & $-1.46(2.80)$ \\
\hline & NIC & $0.68(2.74)$ & $-1.27(3.12)$ \\
\hline \multicolumn{4}{|c|}{ Reactivity (Hotspot imagination) } \\
\hline \multirow[t]{3}{*}{ HR } & $\operatorname{ImRs}$ & $0.38(5.41)$ & $-0.47(4.11)$ \\
\hline & PI & $3.37(5.37)$ & $1.17(4.32)$ \\
\hline & NIC & $3.64(6.60)$ & $1.28(4.17)$ \\
\hline \multirow[t]{3}{*}{ SCL } & ImRs & $-0.33(0.59)$ & $0.67(0.88)$ \\
\hline & PI & $-0.23(0.69)$ & $0.43(0.81)$ \\
\hline & NIC & $-0.60(1.07)$ & $0.66(1.44)$ \\
\hline \multirow[t]{3}{*}{ EMG } & $\operatorname{ImRs}$ & $1.63(2.20)$ & $0.20(3.04)$ \\
\hline & PI & $1.29(3.34)$ & $0.87(3.60)$ \\
\hline & NIC & $2.31(3.62)$ & $0.73(5.07)$ \\
\hline
\end{tabular}

ImRs Imagery Rescripting; PIPositive Imagery; NIC No-Intervention Control Condition; $H R$ mean heartrate in bpm; $S C L$ mean skin conductance level in $\mu \mathrm{S}$; $E M G$ mean Electromyography in $\mu \mathrm{V}$

Reactivity $=$ memory reactivation - baseline

\section{Physiological Outcomes (Hypothesis 4)}

\section{Heart Rate}

Reactivity of HR (Phase 1 and 2) in response to memory retrieval decreased from Session 1 to Session 2, all $F \mathrm{~s}(1$, $72 / 1,70)>9.13, p<0.003, \eta_{p}^{2} s>0.11$, but neither an interaction with condition nor a main effect of condition could be observed, all $F \mathrm{~s}(2,72 / 2,70)<2.60, p>0.081$, $\eta^{2} \mathrm{~s}<0.07$. Descriptive data are provided in Table 4 (Descriptive data of the recovery scores are provided in Table S2, Supplementary Material).

\section{Skin Conductance Level}

For SCL, reactivity (Phase 1 and 2) increased from Session 1 to Session 2, all $F \mathrm{~s}(1,72 / 1,70)>24.09, p<0.001$, $\eta_{\mathrm{p}}^{2} \mathrm{~s}>0.26$, but again no interaction and no significant main effect of condition were evident, all $F \mathrm{~s}(2,72 / 2,70)<1.51$, $p>0.227, \eta_{\mathrm{p}}^{2} \mathrm{~s}<0.04$ (see Table 4).

\section{Electromyography}

EMG reactivity to memory reactivation (Phase 2 ) decreased from Session 1 to Session 2, $F(1,71)=9.51, p=0.003$, $\eta_{p}^{2}=0.12$, but neither an interaction with condition nor a main effect of condition could be observed, all $F \mathrm{~s}(2$, $71)<0.97, p s>0.386, \eta_{p}^{2} s<0.03$.

\section{Discussion}

The present study examined whether ImRs (1) decreases subjective distress as well as state stress symptoms, (2) increases perceived mastery, and (3) reduces subjective emotional and (4) physiological responding to distressing memories compared to a positive imagery (PI) and a no intervention control (NIC) condition.

In line with our first hypothesis and in accordance with results from clinical studies (Morina et al., 2017), ImRs led to significantly stronger reductions of event-related distress during the 1-week follow-up period compared to PI and NIC, indicating that we were able to model therapeutic effects of ImRs treatment. However, ImRs did not lead to a stronger reduction of stress symptoms evoked by the memory reactivation task (i.e. re-experiencing, avoidance, dissociation) as measured with the RSDI, potentially indicating that for less severe life events, habituation occurs after memory reactivation. For the avoidance and dissociation subscale we observed floor effects, which impeded the detection of possible effects of ImRs in the current sample.

Contrary to our second hypothesis, we were not able to replicate the beneficial effects of ImRs on perceived mastery 
(Kunze, Arntz, et al., 2019; Kunze, Lancee, et al., 2019; Strohm et al., 2019), as ImRs was not superior to the control conditions. In the present study, ImRs yielded lower increases in perceived mastery $(d=0.47)$ compared to an earlier study (Strohm et al., 2019: $d=1.17$ ) and a study in individuals with nightmare disorder (Kunze, Arntz, et al., 2019; Kunze, Lancee, et al., 2019). Inconsistent findings might be explained by procedural differences across studies. In the present study, mastery was assessed immediately after participants had reached the hotspot whereas in the study by Strohm et al. (2019) it was obtained after participants had completed the description of the entire sequence of events. In the study by Kunze et al. (2019a, 2019b), mastery was assessed the day after each treatment session with the largest increase of mastery occurring between the first and the second session. These findings might indicate that effects of ImRs on mastery occur later in the therapeutic process. Alternatively, there may have been ceiling effects on mastery as the current sample was rather high-functioning.

The memory reactivation task was successful in activating aversive memories. All distinct negative emotional states assessed (i.e. anxious, angry, sad, guilty, helpless) reached medium to high levels after memory reactivation (see Table S1 in the Supplementary Material). Hypothesis three was partially supported. In line with findings from previous studies (Nilsson et al., 2012; Reimer \& Moscovitch, 2015; Strohm et al., 2019), ImRs led to greater decreases in memory distress than the control conditions. Moreover, participants who received ImRs reported the strongest reductions of helplessness. Sadness also turned out to be a relevant emotion. Although the non-significant interaction seems to suggest that ImRs did not have a specific effect on sadness, the pattern of findings for helplessness and sadness are comparable; however, replication in larger samples is required before firm conclusions can be drawn. For anxiety, anger, guilt, and self-reported arousal no significant group differences emerged. Looking at the life-events reported in the present sample, it seems that anxiety was not the principal emotion related to the memory (see Table S1 in the Supplementary Material), which may explain the lack of treatment effects on anxiety. Significant effects of ImRs on helplessness could either indicate that introducing meaningrelevant changes to the memory by means of ImRs is more beneficial than generating unrelated positive images (as done in PI) or that the effect on helpnessness results from habituation. Future studies should include control conditions also containing exposure to the aversive memory content to rule out alternative explanations. As ImRs was not superior to the control conditions in enhancing perceived mastery and effects on negative emotions were inconsistent, more research is needed to investigate whether reductions in emotional responding are indeed driven by changing negative meanings about mastery.
Regarding hypothesis four, physiological measures differed in their reactivity to the memory reactivation phases. While HR was reactive to both phases, EMG activity increased in response to the hotspot imagination (Phase 2) and SCL in response to the narrative imagination (Phase 1; see Fig. S1-S3 in the Supplementary Material). Contrary to our hypothesis, SCL reactivity increased over time with no differences between groups. This finding can most likely be explained by the fact that baseline SCL scores for Session 1 were found to be unexpectedly high (and significantly higher compared to Session 2) resulting in only small SCL increases when confronted with the memory reactivation task during Phase 1 and even SCL reductions when confronted with Phase 2 (see Fig. S2 in the Supplementary Material) before ImRs. It is probable that within the current study design, we did not allow enough time for SCL to reach resting baseline levels during Session 1 (see Dawson et al., 2007). Furthermore, SCL might also have been increased due to anticipatory arousal to the expected experimental tasks. Consequently, findings for SCL are difficult to interpret with respect to the study hypotheses. For physiological arousal (i.e. HR) and negative emotional valence (i.e. EMG corrugator activity) we found reductions in response to memory reactivation from Session 1 to Session 2 in all groups, indicating that ImRs had no beneficial effects on physiological reactivity over and above habituation effects during the memory reactivation task, which was the same in all conditions. As we used an additional phase of memory reactivation (hotspot imagination), it was not possible to measure the initial physiological response to memory reactivation (i.e. Phase 1 in the present procedure) without participants talking out loud. This procedure was chosen in order to be able to interpret altered physiological responding as resulting from psychological processes and not as a function of changes in respiration patterns produced during speech.

\section{Limitations}

The results of the current study have to be interpreted in light of the following limitations: The ImRs protocol deviated from the standard protocol as used in clinical practice as participants did not switch to an observer perspective. Future studies need to systematically investigate whether viewing the aversive event from the perspective of the younger and the current self is crucial for treatment success or whether changing the sequence of events to a more positive outcome may suffice. Additionally, participants were asked to select a memory from the past two years. While this approach deviates from purely clinical ImRs protocols, it ensures comparability of memory quality and retrieval within the present experimental ImRs framework. Floor effects in the RSDI might result from the fact that life events reported in the 
present study might not have been severe enough to induce high levels of avoidance and dissociation. Furthermore, the timing of the assessment of mastery might not have been ideal and effects might have been diluted by ceiling effects. Moreover, we assessed perceived mastery, memory distress, and emotional states by only one item thereby limiting the reliability of results. Controlling for speech effects on psychophysiology during the first phase of the memory reactivation came at the cost of not having an initial physiological response without participants talking out loud. Finally, our sample only consisted of highly-educated, female participants, therefore results cannot be generalized to the general population and clinical samples.

\section{Conclusions}

The present study showed that ImRs reduces subjectively experienced distress and feelings of helplessness associated with aversive autobiographical memories. The beneficial effects of ImRs on perceived mastery were not replicated in the present study, which might result from timing of its assessment or the fact that a rather high-functioning sample was included. The effects of one brief ImRs session on physiological reactivity did not exceed habituation effects. In order to better understand the mechanisms underlying ImRs, additional research is clearly needed and effects on physiological processes might provide additional insights.

Supplementary Information The online version contains supplementary material available at https://doi.org/10.1007/s10608-021-10233-5.

Acknowledgements The authors kindly thank Milena Aleksic and Johanna Döbig for their support during data collection.

Funding Open Access funding enabled and organized by Projekt DEAL. This research did not receive any funding from agencies in the public, commercial, or not-for-profit sectors.

\section{Declarations}

Conflict of Interest Miriam Strohm, Marena Siegesleitner, Anna E. Kunze, Gabriela G. Werner, Thomas Ehring, \& Charlotte E. Wittekind declare that they have no conflict of interest.

Informed Consent All procedures followed were in accordance with the ethical standards of the responsible committee on human experimentation (national and institutional). Informed consent was obtained from all individual subjects participating in the study.

Animal Rights No animal studies were carried out by the authors for this article.

Open Access This article is licensed under a Creative Commons Attribution 4.0 International License, which permits use, sharing, adaptation, distribution and reproduction in any medium or format, as long as you give appropriate credit to the original author(s) and the source, provide a link to the Creative Commons licence, and indicate if changes were made. The images or other third party material in this article are included in the article's Creative Commons licence, unless indicated otherwise in a credit line to the material. If material is not included in the article's Creative Commons licence and your intended use is not permitted by statutory regulation or exceeds the permitted use, you will need to obtain permission directly from the copyright holder. To view a copy of this licence, visit http://creativecommons.org/licenses/by/4.0/.

\section{References}

American Psychiatric Association (2013). Diagnostic and statistical manual of mental disorders. (5th ed.). American Psychiatric Publishing.

Arntz, A. (2012). Imagery rescripting as a therapeutic technique: Review of clinical trials, basic studies, and research agenda. Journal of Experimental Psychopathology, 3, 189-208. https://doi.org/ $10.5127 /$ jep.024211

Arntz, A., \& Weertman, A. (1999). Treatment of childhood memories: Theory and practice. Behaviour Research and Therapy, 37, 715-740. https://doi.org/10.1016/s0005-7967(98)00173-9

Beda, A., Jandre, F. C., Phillips, D. I., Giannella-Neto, A., \& Simpson, D. M. (2007). Heart-rate and blood-pressure variability during psychophysiological tasks involving speech: Influence of respiration. Psychophysiology, 44, 767-778. https://doi.org/10.1111/j. 1469-8986.2007.00542.x

Blechert, J., Peyk, P., Liedlgruber, M., \& Wilhelm, F. H. (2016). ANSLAB: Integrated multichannel peripheral biosignal processing in psychophysiological science. Behavior Research Methods, 48, 1528-1545. https://doi.org/10.3758/s13428-015-0665-1

Bradley, M. M., \& Lang, P. J. (1994). Measuring emotion: The SelfAssessment Manikin and the semantic differential. Journal of Behavior Therapy and Experimental Psychiatry, 25, 49-59. https://doi.org/10.1016/0005-7916(94)90063-9

Çili, S., Pettit, S., \& Stopa, L. (2016). Impact of imagery rescripting on adverse self-defining memories and post-recall working selves in a non-clinical sample: A pilot study. Cognitive Behaviour Therapy, 46, 75-89. https://doi.org/10.1080/16506073.2016.1212396

Craske, M. G., Kircanski, K., Zelikowsky, M., Mystkowski, J., Chowdhury, N., \& Baker, A. (2008). Optimizing inhibitory learning during exposure therapy. Behaviour Research and Therapy, 46, 5-27. https://doi.org/10.1016/j.brat.2007.10.003

Dawson, M. E., Schell, A. M., \& Filion, D. L. (2007). The Electrodermal System. In J. Cacioppo, L. G. Tassinary, \& G. G. Berntson (Eds.), The Handbook of Psychophysiology. (pp. 159-181). Cambridge University Press. https://doi.org/10.1017/CBO9780511 546396.007

Dibbets, P., Lemmens, A., \& Voncken, M. (2018). Turning negative memories around: Contingency versus devaluation techniques. Journal of Behavior Therapy and Experimental Psychiatry, 60, 5-12. https://doi.org/10.1016/j.jbtep.2018.02.001

Dibbets, P., Poort, H., \& Arntz, A. (2012). Adding imagery rescripting during extinction leads to less ABA renewal. Journal of Behavior Therapy and Experimental Psychiatry, 43, 614-624. https://doi. org/10.1016/j.jbtep.2011.08.006

Ehlers, A., \& Clark, D. M. (2000). A cognitive model of posttraumatic stress disorder. Behaviour Research and Therapy, 38, 319-345. https://doi.org/10.1016/S0005-7967(99)00123-0

Foa, E. B., \& Kozak, M. J. (1986). Emotional processing of fear: Exposure to corrective information. Psychological Bulletin, 99, 20-35. https://doi.org/10.1037/0033-2909.99.1.20

Foa, E. B., \& Rothbaum, B. O. (1998). Treating the trauma of rape: Cognitive behavioral therapy for PTSD. Guilford Press. 
Foa, E. B., Steketee, G., \& Rothbaum, B. O. (1989). Behavioral/cognitive conceptualizations of post-traumatic stress disorder. Behavior Therapy, 20, 155-176. https://doi.org/10.1016/S0005-7894(89) 80067-X

Fridlund, A. J., \& Cacioppo, J. T. (1986). Guidelines for human electromyographic research. Psychophysiology, 23, 567-589. https:// doi.org/10.1111/j.1469-8986.1986.tb00676.x

Hackmann, A. (2011). Imagery rescripting in posttraumatic stress disorder. Cognitive and Behavioral Practice, 18, 424-432. https:// doi.org/10.1016/j.cbpra.2010.06.006

Hagenaars, M. A., \& Arntz, A. (2012). Reduced intrusion development after post-trauma imagery rescripting: An experimental study. Journal of Behavior Therapy and Experimental Psychiatry, 43, 808-814. https://doi.org/10.1016/j.jbtep.2011.09.005

Holmes, E. A., Arntz, A., \& Smucker, M. R. (2007). Imagery rescripting in cognitive behaviour therapy: Images, treatment techniques and outcomes. Journal of Behavior Therapy and Experimental Psychiatry, 38, 297-305. https://doi.org/10.1016/j.jbtep.2007. 10.007

Hopper, J. W., Frewen, P. A., Sack, M., Lanius, R. A., \& van der Kolk, B. A. (2007). The responses to script-driven imagery scale (RSDI): Assessment of state posttraumatic symptoms for psychobiological and treatment research. Journal of Psychopathology and Behavioral Assessment, 29, 249-268. https://doi.org/10.1007/ s10862-007-9046-0

Hyett, M. P., Bank, S. R., Lipp, O. V., Erceg-Hurn, D. M., Alvares, G. A., Maclaine, E., \& McEvoy, P. M. (2018). Attenuated psychophysiological reactivity following single-session group imagery rescripting versus verbal restructuring in social anxiety disorder: Results from a randomized controlled trial. Psychotherapy and Psychosomatics, 87, 340-349. https://doi.org/10.1159/000493897

Krönke, K., Spitzer, R. L., \& Williams, J. B. W. (2001). The PHQ-9: Validity of a brief depression severity measure. Journal of General Internal Medicine, 16, 606-613. https://doi.org/10.1046/j. 1525-1497.2001.016009606.x

Kunze, A. E., Arntz, A., \& Kindt, M. (2019). Investigating the effects of imagery rescripting on emotional memory: A series of analogue studies. Journal of Experimental Psychopathology. https:// doi.org/10.1177/2043808719850733

Kunze, A. E., Lancee, J., Morina, N., Kindt, M., \& Arntz, A. (2019). Mediators of change in imagery rescripting and imaginal exposure for nightmares: Evidence from a randomized wait-list controlled trial. Behavior Therapy, 50, 978-993. https://doi.org/10.1016/j. beth.2019.03.003

Lang, P. J. (1979). A bio-informational theory of emotional imagery. Psychophysiology, 169, 495-512. https://doi.org/10.1111/j.14698986.1979.tb01511.x

Löwe, B., Spitzer, R. L., Zipfel, S. \& Herzog, W. (2002). Gesundheitsfragebogen für Patienten (PHQ-D). Komplettversion und
Kurzform (2. Auflage) [Patient Health Questionnaire (PHQ-D). Complete and short version]. Pfizer: Karlsruhe.

Mauss, I. B., \& Robinson, M. D. (2009). Measures of emotion: A review. Cognition and Emotion, 23, 209-237. https://doi.org/10. 1080/02699930802204677

Morina, N., Lancee, J., \& Arntz, A. (2017). Imagery rescripting as a clinical intervention for aversive memories: A meta-analysis. Journal of Behavior Therapy and Experimental Psychiatry, 55, 6-15. https://doi.org/10.1016/j.jbtep.2016.11.003

Nilsson, J., Lundh, L., \& Viborg, G. (2012). Imagery rescripting of early memories in social anxiety disorder: An experimental study. Behaviour Research and Therapy, 50, 387-392. https://doi.org/10. 1016/j.brat.2012.03.004

Quintana, D. S., \& Heathers, J. A. J. (2014). Considerations in the assessment of heart rate variablitiy in biobehavioral research. Frontiers in Psychology, 5, 805. https://doi.org/10.1016/10.3389/ fpsyg.2014.00805

Reimer, S. G., \& Moscovitch, D. A. (2015). The impact of imagery rescripting on memory appraisals and core beliefs in social anxiety disorder. Behaviour Research and Therapy, 75, 48-59. https:// doi.org/10.1016/j.brat.2015.10.007

Sheehan, D. V. (2016). Mini International Neuropsychiatric Interview 7.0.2. Jacksonville, FL: Medical Outcomes Systems.

Sheehan, D. V., Lecrubier, Y., Sheehan, K. H., Amorim, P., Janavs, J., Weiller, E., et al. (1998). The Mini-International Neuropsychiatric Interview (MINI): the development and validation of a structured diagnostic psychiatric interview for DSM-IV and ICD-10. Journal of Clinical Psychiatry, 59(suppl 20), 22-33.

Strohm, M., Siegesleitner, M., Kunze, A. E., Ehring, T., \& Wittekind, C. E. (2019). Imagery Rescripting of aversive autobiographical memories: Effects on memory distress, emotions, and feelings of mastery. Cognitive Therapy and Research, 43, 1005-1017. https:// doi.org/10.1007/s10608-019-10021-2

Tolgou, T., Rohrmann, S., Stockhausen, C., Krampen, D., Warnecke, I., \& Reiss, N. (2018). Physiological and psychological effects of imagery techniques on health anxiety. Psychophysiology, 55, 1-12. https://doi.org/10.1111/psyp.12984

Wilhelm, F. H., Grossman, P., \& Roth, W. T. (1999). Analysis of cardiovascular regulation. Biomedical Sciences Instrumentation, 35, 135-140.

Publisher's Note Springer Nature remains neutral with regard to jurisdictional claims in published maps and institutional affiliations. 